\title{
The Bulge-disc connection in the Milky Way
}

\author{
James Binney \\ Rudolf Peierls Centre for Theoretical Physics, \\ Keble Road, \\ Oxford OX1 3NP, UK \\ email: binney@thphys.ox.ac.uk
}

\begin{abstract}
Bulges come in two flavours - classical and pseudo. The principal characteristics of each flavour are summarised and their impact on discs is considered. Classical bulges probably inhibit the formation of stellar discs. Pseudobulges exchange angular momentum with stars and gas in their companion discs, and also with its embedding dark halo. Since the structure of a pseudobulge depends critically on its angular momentum, these exchanges are expected to modify the bulge. The consequences of this modification are not yet satisfactorily understood. The Galaxy has a pseudobulge. I review the manifestations of its interaction with the disc. More work is needed on the dynamics of gas near the bulge's corotation radius, and on tracing the stellar population in the inner few hundred parsecs of the Galaxy.

Galaxy: kinematics and dynamics; Galaxy: bulge; galaxies: kinematics and dynamics
\end{abstract}

\section{Introduction}

In the last decade a consensus has formed that there are two types of bulges: classical bulges, which resemble low-luminosity elliptical galaxies, and "pseudobulges", which do not - for an authoritative case for this distinction see Kormendy \& Kennicutt (2004), and for an update on the evidence see Bureau et al. (2008). It is believed that pseudobulges form through dynamical instabilities of discs, while classical bulges are products of major mergers. The rapid fluctuations of the gravitational field during a merger randomises the orbits of stars from the progenitor galaxies and violently shocks the progenitors' gas. Stars form rapidly in this gas at a time when there are no circular orbits, so the the stellar system formed is not a centrifugally supported disc but a hot stellar system. Thus classical bulges are highly chaotic stellar systems. A pseudobulge also forms when the gravitational field is unsteady, but the field is not as chaotic as during a major merger, and the pseudobulge inherits a more ordered phase-space structure from its progenitor disc. In particular, it settles to a more rapidly rotating configuration than does a classical bulge, and it is usually triaxial.

In this review I discuss the interactions between bulges of both types and discs, and examine more particularly the case of the Milky Way. The bulge of our Galaxy is still rather mysterious. From our vantage point near the edge of the optical disc, most of it is heavily obscured, and from our edge-on perspective it would be non-trivial to disentangle the bulge's three-dimensional structure even if we had a clear field of view. However, there is every indication that our bulge is a pseudobulge. For this reason alone it would be connected to the disc by history. The connection between bulge and disc can be seen to be ongoing also.

\section{Classical bulges}

If we define classical bulges to be the analogues of low-luminosity elliptical galaxies, which appear to be axisymmetric (Cappellari et al. 2007), then they will be axisymmetric 


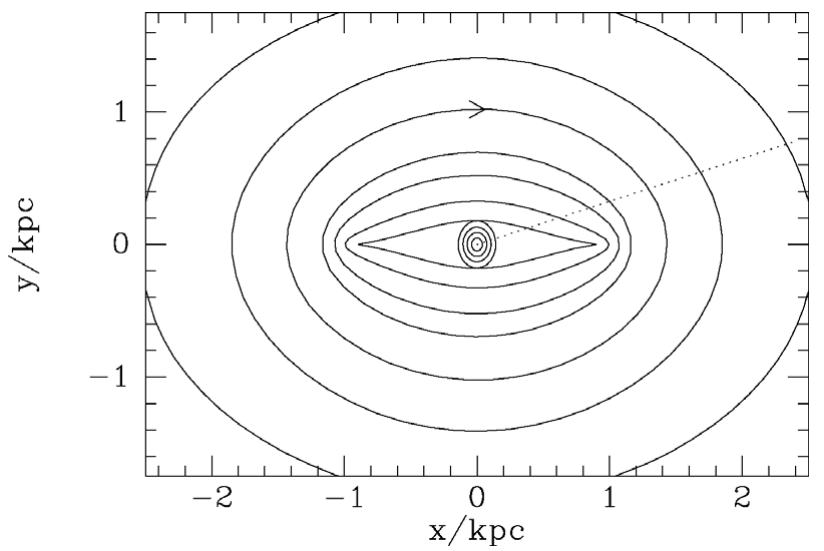

Figure 1. Orbits in a rotating barred potential viewed in the potential's rotating frame. The central orbit family forms the $x_{2}$ disc, while the larger, elongated orbits belong to the $x_{1}$ family (from Binney \& Merrifield 1998).

too. Consequently, we do not expect classical bulges to exchange angular momentum with discs.

Since a thin stellar disc cannot survive a major merger, classical bulges have to be older than any disc that surrounds them. The extraordinary S0 galaxy NGC 4550, which contains two co-spatial discs that rotate in opposite senses (Rubin et al. 1992), is a clear indication that galaxies can experience distinct episodes of gas accretion, and that the angular momentum vectors of the gas accreted in the different episodes can be seriously misaligned. Presumably disc galaxies with classical bulges arise when a spheroid that formed in a merger experiences an episode of gas accretion.

Rix \& White (1990) showed that many "elliptical" galaxies could host stellar discs. The formation of such faint discs is an ill-understood problem. There are several ways in which the dominant spheroid could profoundly modify the formation of a disc:

- Strong shear associated with the large and fairly constant circular speed generated by the spheroid will inhibit gravitational instability on the gas disc and make any spiral structure tightly wound.

- UV radiation from the spheroid's stars will have a tendency to photo-dissociate molecules, thus increasing the column density of gas required for the transition from atomic to molecular gas that must precede the formation of the cool interstellar cores within which stars form.

- Massive spheroids have a tendency to fill up with gas at the system's virial temperature, $T_{\text {vir }}$. This tendency is a consequence of (a) the depth of the gravitational potential well of a massive spheroid, and (b) the large random velocities of the spheroid's stars, which lead to winds from AGB stars colliding at high speed and shocking to $T_{\text {vir }}$. The depth of the potential well is significant because it controls whether supernova-heated gas flows out of the galaxy as a wind or accumulates as an X-ray emitting atmosphere. A dense hot atmosphere has the potential to ablate and heat cold gas, thus terminating star formation in that system. I have argued elsewhere (Binney 2004; Nipoti \& Binney 2007) that this process is responsible for the cutoff in the galaxy luminosity function at the Schechter luminosity $L_{*}$ and the transfer of galaxies to the red sequence.

Thus an important and still insufficiently understood area of bulge/disc interaction is the suppression of disc growth by luminous classical bulges. 


\section{Pseudobulges}

Pseudobulges are recognised by their low Sersic indices (their radial surface-bright profiles are closer to exponentials than $r^{1 / 4}$-laws), fast rotation, and triaxiality (Kormendy $\&$ Kennicutt 2004). In earlier-type pseudobulges the mass distribution is sufficiently centrally concentrated to support an inner Lidblad resonance (ILR) (Athanassoula 1992). Inside the ILR the orbits are only mildly non-circular and elongated perpendicular to the bar (Fig. 1). Gas can accumulate on these orbits, and in many systems is dense enough to be support a ring of vigorous star formation. Consequently, pseudobulges, unlike classical bulges, often contain many young stars.

The orbits whose vertical instability drives the formation of a pseudobulge dominate the density at a fair fraction of $R_{\mathrm{CR}}$ (Pfenniger \& Friedli 1991). Consequently, this is the radial range over which a pseudobulge is vertically thick. Further out, around corotation, in the region of the $x_{2}$ disc, the system remains fairly thin vertically (Athanassoula 2005). Thus rather counter-intuitively, important parts of a pseudobulge are disc-like. In particular, the sharp upward rise in the brightness profile of a face-on pseudobulge is more likely to be due to a thin luminous $x_{2}$ disc than to a bulge in the classical sense.

Gas that between corotation and the $x_{2}$ disc cannot follow nearly circular orbits. At the outer edge of this region gas may flow roughly along $x_{1}$ orbit (Fig. 1), but shocks tend to develop in the flow, which deprive the gas of energy, so it drifts inwards at a nonnegligible rate, surrendering angular momentum to the stellar bar as it goes. If there is an $x_{2}$ disc, the shocks are displaced from the long axis of the bar so as to nearly touch the intersection of the edge of the $x_{2}$ disc and the galaxy's minor axis (Athanassoula 1992). In the absence of an $x_{2}$ disc, the shocks keep close to the bar's major axis. The shocks are displaced from the major axis in the downstream direction, and the enhancement of the gas density in the post-shock region causes the bar's gravitational field to drain angular momentum from the gas. At optical wavelengths the shocks manifest themselves as dust lanes. The speed with which shocks drain energy and angular momentum from gas in the region $R_{\mathrm{CR}}>R>R_{\mathrm{ILR}}$ causes the surface density of gas in this region to be small relative to the density outside corotation and in the $x_{2}$ disc.

\subsection{Impact on spiral structure}

Self-sustaining spiral disturbances in a centrifugally supported disc occur either inside their corotation radius (as in the classical WKBJ picture - see Binney \& Tremaine 2008, $\S 6.3$ ) or in the immediate vicinity of this radius (Sellwood \& Kahn 1991). Since the corotation radius of the bar of a spiral galaxy lies near the ends of the bar, it follows that self-sustaining spiral disturbances rotate less rapidly that the bar; in most of the disc the spiral pattern cannot rotate with the bar (Sellwood \& Sparke 1988). However, strong forcing of the disc near the end of the bar can produce a corotating spiral disturbance.

\subsection{Exchanges of angular momentum}

We have seen that inside $R_{\mathrm{CR}}$ gas surrenders angular momentum to the bar. Gas and stars outside $R_{\mathrm{CR}}$ tend to take up angular momentum from the bar. In particular, gas piles up outside $R_{\mathrm{CR}}$ as gas spiralling inward from the outer disc acquires angular momentum from the bar as it approaches $R_{\mathrm{CR}}$. Over time stars formed in this region of enhanced gas density form a stellar ring. A further stellar ring often develops further out in the region of the outer Lindblad resonance (OLR), where the orientation of near-circular closed orbits changes from aligned with the bar (inside the OLR) to aligned perpendicular to the bar. These rings have been used to infer the pattern speeds of the bars (Buta \& Combes 1996). 


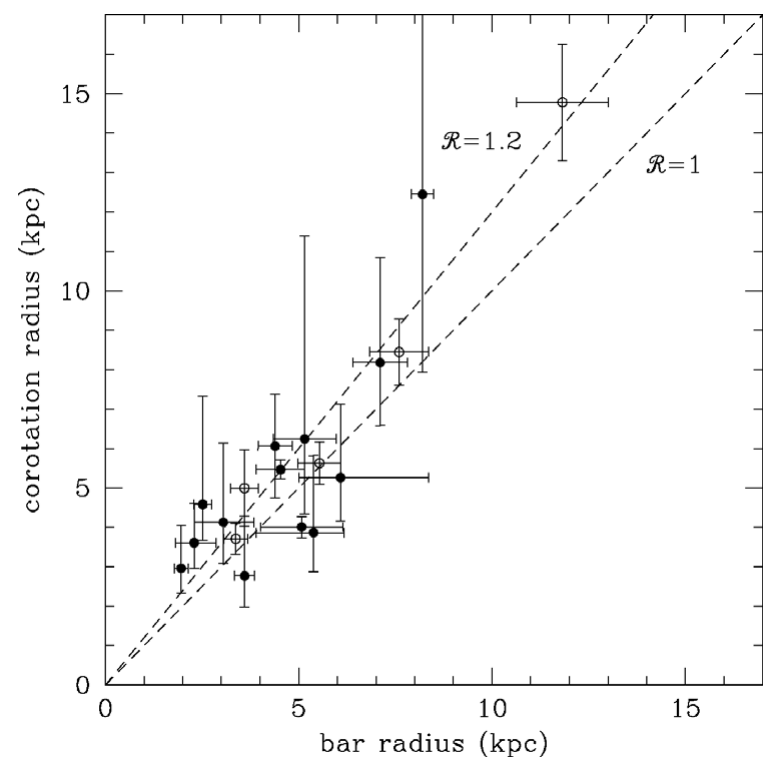

Figure 2. Measurents of corotation radius $R_{\mathrm{CR}}$ and bar semi-axis length $a$ from a variety of sources. The straight lines show constant values of $\mathcal{R}=R_{\mathrm{CR}} / a$ (From Binney \& Tremaine 2008).

\subsection{Impact on the bar}

A bar consists of orbits that have less angular momentum than circular orbits with the same energy. In this sense, bars are negative-angular momentum perturbations of centrifugally supported discs. The pattern speed of the bar is set by the mean precession speed of the elliptical orbits of its stars. When an orbit of the bar loses angular momentum, it becomes more elongated and precesses more slowly. Consequently, when the bar loses angular momentum, it becomes stronger and slower, and conversely when it gains angular momentum. In particular, gas in the region $R_{\mathrm{OLR}}<R<R_{\mathrm{CR}}$ weakens and accelerates the bar as it spirals in, while when gas and stars outside $R_{\mathrm{CR}}$ take up angular momentum from the bar, they strengthen and slow it. Bars will also lose angular momentum to a slowly on non-rotating dark halo on a timescale that is not long compared to the Hubble time (Weinberg \& Tremaine 1984; Debattista \& Sellwood 1998).

Numerical simulations of bars forming from unstable discs suggests that all bars are born "fast", that is they have $\mathcal{R} \equiv R_{\mathrm{CR}} / a \simeq 1.2$. The empirical evidence that real bars are fast is shown in Fig. 2. This finding suggests that losses of angular momentum by the bar to the dark halo and the disc at $R>R_{\mathrm{CR}}$ is balanced by gains of angular momentum from gas that makes it through the barrier at $R_{\mathrm{CR}}$. Certainly there is the possibility of stabilising feedback in that when a bar loses angular momentum, $R_{\mathrm{CR}}$ increases, so the non-axisymmetric forces on gas near $R_{\mathrm{CR}}$ weaken. Since it is these forces that must be overcome by dissipation if gas is to cross $R_{\mathrm{CR}}$, a decrease in the bar's pattern speed will increase the rate at which gas crosses $R_{\mathrm{CR}}$, and this increase will in turn drive the pattern speed back up.

This proposal, that the pattern speeds of bars are kept up by gas that passes $R_{\mathrm{CR}}$, would appear not to apply to gas-poor S0 galaxies, and in fact most measured pattern speeds are for such systems. Therefore these measurements are currently puzzling.

Bournaud \& Combes (2002) propose a more radical explanation. By bringing mass towards the centre, bars endanger their health: once the potential becomes sufficiently centrally concentrated, the bar dissolves (Hasan \& Norman 1990; Friedli \& Benz 1993). 
Perhaps after a bar forms, inspiralling gas builds up outside $R_{\mathrm{CR}}$, while the gas that was initially inside $R_{\mathrm{CR}}$ flows to the centre and weakens the bar. Eventually the bar is destroyed and the gas waiting outside $R_{\mathrm{CR}}$ flows inwards, and reaches a sufficient surface density to form a new bar. In their numerical simulations Bournaud \& Combes (2002) foud that the process of gradual weakening of the bar followed by death and the inward rush of new material can repeat three times in a Hubble time. Each new bar was smaller and faster than the previous one.

\section{Case of the Milky Way}

There is much evidence that the Milky Way possesses a pseudobulge:

- The COBE/DIRBE near-IR photometry of the bar shows the characteristic "peanut" shape of a pseudobulge.

- Photometry of individual sources, such as clump giants, shows that at longitudes $l \sim 5^{\circ}$ the bulge is about $0.3 \mathrm{mag}$ closer than at $l \sim-5^{\circ}$ (Stanek et al. 1997). Thus the bulge is triaxial and and the nearer end is on our left.

- The longitude-velocity plots of both HI and CO show many features indicative of gas flow in a barred potential. The most prominent is the swath of $\mathrm{CO}$ emission that runs diagonally across the $(l, v)$ plot for CO, from $\left(l \simeq 30^{\circ}, v \simeq 100 \mathrm{~km} \mathrm{~s}^{-1}\right)$ to the equivalent point on the other side of the origin (Dame et al. 2001). This is the signature of a ring of gas of radius $R_{0} \sin (30) \simeq 4 \mathrm{kpc}$. At $l>0$ and velocities greater than those of this band there is a marked dearth of gas until $l$ drops to a couple of degrees. This is the signature of the almost empty region between $R_{\mathrm{CR}}$ and the edge of the $x_{2}$ disc at $\sim R_{0} \sin (1.8 \mathrm{deg}) \simeq 250 \mathrm{pc}$. At $-1 \lesssim l \lesssim 1.8^{\circ}$ there is a ridge of emission by CS (which traces very dense gas) at velocities that extend up to $\sim 100 \mathrm{~km} \mathrm{~s}^{-1}$ (Binney et al. 1991). This is the signature of the $x_{2}$ disc. Another important feature is the narrow band of strong gas emission that slopes downwards to the right in $(l, v)$ plots, crossing $l=0$ at $v \simeq-53 \mathrm{~km} \mathrm{~s}^{-1}$ and reaching the curve of tangent velocities at $l \simeq-22^{\circ}$. This "expanding $3 \mathrm{kpc}$ arm" (van Woerden et al. 1957) is probably associated with the ultraharmonic (4:1) resonance just inside $R_{\mathrm{CR}}$. In this interpretation the arm lies on the near side of the centre. Dame \& Thaddeus (2008) have recently identified the counterpart on the far side, and conclude that this feature is satisfyingly symmetrical to the near-side arm. Interestingly they are in the plane $b=0$ whereas much of the HI on $x_{1}$ orbits appears to be in a plane that is significantly inclined to this plane (Liszt \& Burton 1980; Ferrière et al. 2007).

The Sun is expected to lie near the OLR of the bar. Kalnajs (1991) pointed out that in this region the local stellar velocity distribution might be expected to be bimodal: at a resonance the orientation of the closed orbits shifts through $90^{\circ}$ and near the resonance one would expect to find stars trapped around both kinds of closed orbit, with the result that the velocity distribution would be bimodal. Subsequently Raboud et al. (1998) and Fux (1999) used this effect to explain the distribution of velocities in the Hipparcos catalogue. Dehnen (2000) simulated the effect on the local velocity distribution of the adiabatic growth of the bar potential. He found that the simulated velocity distributions resemble the Hipparcos distribution only if the Sun lies outside the bar's OLR $\left(R_{\mathrm{OLR}} \simeq\right.$ $\left.0.85 R_{0}\right)$. The crucial feature is the Hercules stream, which is very prominent in the Hipparcos catalogue and made up of stars that are moving outwards on on orbits that are aligned with the bar. From this analysis Dehnen obtains a value for the pattern speed of the bar $\left[(1.85 \pm 0.15) \Omega\left(R_{0}\right)\right]$ that agrees well with independent estimates, for example that obtained by simulating the structure of the $(l, v)$ diagram of molecular gas (Englmaier \& Gerhard 1999). 
Near-IR star counts from the 2-MASS (Hammersley et al. 2000) and TCS-CAIN catalogues (Cabrera-Lavers et al. 2007) and Spitzer data (Benjamin et al. 2005) show overdensities of stars within $100 \mathrm{pc}$ of the plane at longitudes satisfying $20^{\circ}<l<27^{\circ}$ and distances that imply galactocentric radii $3-4.5 \mathrm{kpc}$. These features, which include luminous, presumably young stars, seem to lie around or just outside $R_{\mathrm{CR}}$ for the bar along a line through the Galactic centre that is inclined to the Sun-centre line by $\sim 43^{\circ}$. This inclination lies outside the range $30^{\circ}-15^{\circ}$ favoured by studies of both the COBE/DIRBE photometry (Binney et al. 1997; Bissantz \& Gerhard 2002) and the flow of gas in the bar's potential (Fux 1999; Englmaier \& Gerhard 1999). Analyses of the COBE/DIRBE photometry concentrate on the sky at $b \gtrsim 2^{\circ}$ to minimise the effects of obscuration, so they would not be sensitive to the overdensities in the near-IR star counts.

Hammersley et al. (2000) suggested that these features form "a long thin bar", but it is not clear that the features extend to the centre. It might make more sense dynamically if the features were confined to the corotation region, when they might be created in the disc by the bar's forcing. It would remain puzzling that they lie on the leading edge of the bulge/bar. We still have some way to go before we have a coherent picture of the Galaxy's structure in this region of strongest interaction between the bulge and the disc.

Do we see evidence that the bulge is related to the disc, as the standard theory of pseudobulge formation implies? There does seem to be a strong connection between the bulge and the thick disc. Both systems are old (e.g. McWilliam \& Rich 1994; Zoccali et al. 2006) and have similar star-formation histories as diagnosed by the distributions of their stars in the $([\mathrm{O} / \mathrm{Fe}],[\mathrm{Fe} / \mathrm{H}])$ plane (Meléndez et al. 2008). Haywood (2008) has argued effectively that the chemical evolution of the thin disc follows on naturally from the end point of thick-disc formation. Hence at the moment the data are all consistent with the conjecture that the bulge and thick disc formed from the Galaxy's early disc, and the thin disc has gradually accumulated in the long quiet period that followed. The major caveat that should be made here is that the $x_{2}$ disc is clearly the site of vigorous star formation, as attested, for example, by the numerous supernova remnants detected in the central $100 \mathrm{pc}$ at radio continuum wavelengths (LaRosa et al. 2000). In external galaxies similar to the Milky Way central star-forming discs and rings are often prominent and significantly affect our characterisation of the whole bulge. In the case of the Galaxy this region is so highly obscured at optical wavelengths that it has had no impact on our characterisation of the bulge. For example, we say that the bulge is old because the stellar population that is studied a few degrees off the plane is old. When technology allows us to determine the ages and abundances of stars that lie near the plane at $r<250 \mathrm{pc}$, we will probably reassess the bulge. If the $x_{2}$ disc has been forming stars throughout the life of the Galaxy, as is perfectly likely, it would be interesting to know how these stars are distributed now.

\section{Summary}

Bulges fall into two classes: classical and pseudobulges. The impact that a classical bulge has on the associated disc is still speculative, but there is a distinct possibility that a luminous classical bulge will suppress the formation of a co-spatial stellar disc.

Pseudobulges are fashioned out of a disc that has gone bar unstable. Because their patterns rotate, they inevitably exchange angular momentum with any other nearby component.

Bars weaken and speed up when they gain angular momentum and strengthen and slow down when they lose it. It is natural for a bar to lose angular momentum to the disc that lies beyond $R_{\mathrm{CR}}$ and to the dark halo. The loss of angular momentum to gas 
in the surrounding disc leads to a buildup of gas outside $R_{\mathrm{CR}}$ and the formation thee of an "inner" stellar ring. "Outer" rings can also form at the outer Lindblad resonance of the bar.

Gas that makes it across the barrier around $R_{\mathrm{CR}}$ quickly surrenders most of its angular momentum and settles onto the $x_{2}$ disc if one exists. The buildup of gas on the $x_{2}$ disc frequently leads to rapid star formation and the formation of a "nuclear" stellar ring.

All the bars that have had their pattern speeds measured to date prove to be "fast" rotators in the sense that their corotation radius lies within $\sim 1.2$ of their ends. This finding is remarkable because the timescale for bars to gain or lose angular momentum is thought to be significantly shorter than the Hubble time. In spiral galaxies one might conjecture that there is a balance between the angular-momentum gain from gas that slips past $R_{\mathrm{CR}}$ and angular-momentum loss to the outer stellar disc and the dark halo. However, this conjecture does not explain why the bars of S0 galaxies, which contain little gas, are also fast. An alternative explanation is that bars destroy themselves by bringing gas in and making the galactic potential more centrally concentrated. After the bar has self-destructed, gas accumulates on circular orbits in the bar's old radial range, and forms a new bar once it has reached a critical mass.

Our Galaxy's bulge displays all the characteristics of a pseudobulge: above the plane we see the typical peanut shape of the thick part of the bar, and in the plane we see a star-forming $x_{2}$ disc, a deficiency of gas between this disc and a ring of gas that lies outside $R_{\mathrm{CR}}$. We also see features in the local velocity distribution that are attributable to the bar's OLR lying just inside $R_{0}$. The first indications that the Galaxy is barred were the presence of gas with large radial velocities along the line of sight to the Galactic centre. The biggest concentration of such gas lies where the " $3 \mathrm{kpc}$ " arm crosses the SunCentre line. Recently its counterpart on the far side of the Galaxy has been identified. It is remarkably similar to the near-side arm, as we would expect of a feature that is driven by a bar rather than spiral structure. These arms probably lie at the ultraharmonic $(4: 1)$ resonance, but this has yet to be securely established.

Although the idea of a Galactic bar is able to account for a large number of observations in a satisfying way, there is still one major puzzle to resolve. This is the status of overdensities in the near-IR starcounts that have been interpreted in terms of a "longthin bar." These overdensities are securely established at larger longitudes than those at which we expect to see the end of the conventional bar, and photometric distance estimates to these features suggest that they lie along a line through the Galactic centre that is rotated in the direction of Galactic rotation by $15-20 \mathrm{deg}$ with respect to the long axis of the bulge/bar. Since the overdensities are confined to $\sim 100 \mathrm{pc}$ of the plane and involve luminous stars, they are presumably associated with gas flow. More work needs to be done on the flow of gas around the Galaxy in the range $5-3 \mathrm{kpc}$.

The stellar population of the readily observed thick part of the bar is remarkably similar to that of the thick disc. This finding suggests that these two components formed simultaneously from an early, gas-rich and dynamically unstable thin disc. In addition to the old stellar bulge population that has been studied to date, the central bulge must contain a younger population of stars formed in the $x_{2}$ disc. Finding these stars and deducing the star-formation history of the $x_{2}$ disc is an important task for the future.

\section{References}

Athanassoula, E. 1992, MNRAS, 259, 328

Athanassoula, E. 2003, MNRAS, 341, 1179

Athanassoula, E. 2005, MNRAS, 358, 1477 
Benjamin, R. A., et al. 2005, ApJ, 630, L149

Binney, J., Gerhard, O. E., Stark, A. A, Bally, J., \& Uchida, K. I. 1991, MNRAS, 252, 210

Binney, J., Gerhard, O. E., \& Spergel, D. 1997, MNRAS, 288, 365

Binney J. \& Merrifield, M. 1998, Galactic Astronomy (Princeton: Princeton University Press)

Binney, J. 2004, MNRAS, 347, 1093

Binney J. \& Tremaine, S. 2008, Galactic Dynamics (Princeton: Princeton University Press)

Bissantz, N. \& Gerhard, O. E. 2002, MNRAS, 330, 591

Block, D.L., Bournaud, F., Combes, F., Puerari, I. \& Buta, R. 2002, A\&A, 394, L35

Combes, F. \& Sanders, R. H. 1981, A\&A, 96, 164

Bournaud, F. \& Combes, F. 2002, A\&A, 392, 83

Bureau M., Athanassoula, E., \& Barbuy, B. 2008, Proc.Intl.Astron.U., 3, IAU Symp. 245

Buta, R. \& Combes, F. 1996, FCPh, 17, 95

Cabrera-Lavers, A., Hammersley, P. L., González-Fernández, C., López-Corredoira, M., Garzón, F., \& Mahoney, T. J. 2007, A\&A, 465, 825

Cappellari, M., \& the SAURON collaboration, 2007, MNRAS, 379, 418

Dame, T. M., Hartmann, Dap., \& Thaddeus, P. 2001, ApJ, 547, 792

Dame, T. M. \& Thaddeus, P. 2008, ApJL, in press (arXiv:0807.1752)

Debattista, V. P. \& Sellwood, J. A. 1998, ApJ, 493, L5

Dehnen, W. 2000, AJ, 119, 800

Englmaier, P. \& Gerhard, O. 1999, MNRAS, 304, 512

Ferrière, K., Gillard, W. \& Jean, P. 2007, A\&A, 467, 611

Friedli, D. \& Benz, W., A\&A, 268, 65

Fux, R. 1999, A\&A, 345, 787

Hammersley, P. L., Garzon, F., Mahoney, T. J., Lopez-Corredoira, M., \& Torres, M. A. P. 2000, MNRAS, 317, L45

Hasan, H. \& Norman, C. A. 1990, ApJ, 361,69

Haywood, M. 2008, arXiv:0805.1822

Kalnajs, A. 1991, in "Dynamics of Disc Galaxies", ed. B. Sundelius (Göteborg: Dept of Astron. Astrophys., Göteborg Univ.), 323

Kormendy, J. \& Kennicutt, R. 2004, ARA\&A, 42, 603

LaRosa, T. N., Kassim, N. E., Lazio, T. J. W., \& Hyman, S. D., 2000, AJ, 119, 207

Liszt, H. S. \& Burton, W. B. 1980, ApJ, 236, 779

McWilliam, A. \& Rich, R. M. 1994, ApJS, 91, 749

Meléndez, J., et al. 2008, A\&A, 484, L21

Nipoti, C. \& Binney, J. 2007, MNRAS, 382, 1481

Pfenniger D. \& Friedli D. 1991, A\&A, 252, 75

Raboud, D., Grenon, M., Martinet, L., Fux, R. \& Udry, S. 1998, A\&A, 335, L61

Raha, N., Sellwood, J. A., James, R. A. \& Kahn, F. D., Nature, 352, 411

Rix H.-S. \& White S. D. M. 1990, ApJ, 362, 52

Rubin, V. C., Graham, J. A., \& Kenney, J. D. P. 1992, ApJ, 394, L9

Sellwood, J. A. \& Kahn, F. 1991, MNRAS, 250, 278

Sellwood, J. A. \& Sparke, L. S. 1988, MNRAS, 231, P25

Stanek, K. Z., Udalski, A., Szymanski, M., Kaluzny, J., Kubiak, M., Mateo, M. \& Krezeminski, W. 1997, ApJ, 477, 163

van Woerden, H., Rougoor, G. W., \& Oort, J. H. 1957, Comp. Rend., 244, 1691

Weinberg, M. D. \& Tremaine, S. 1984, MNRAS, 209, 729

Zoccali, M., et al. 2006, A\&A, 457, L1 